\title{
Oral Manifestations and Diabetes
}

\author{
Nélio Veiga*1,2, Tiago Marques ${ }^{1,2}$, Ana Sofia Monteiro ${ }^{1}$, João Couto ${ }^{1}$, Pedro Conceição ${ }^{1}$, Sara Matos ${ }^{1}$, Joana Santos ${ }^{1}$, \\ Ana Raquel Pereira ${ }^{1,}$ Sarah Goolamhussen ${ }^{1}$, Rita Grão ${ }^{1}$, Marta André ${ }^{1}$ and Inês Coelho ${ }^{3}$ \\ ${ }^{1}$ Health Sciences Institute, Universidade Católica Portuguesa, Portugal \\ ${ }^{2}$ Centre for Interdisciplinary Health Research (CIIS), Universidade Católica Portuguesa, Portugal \\ ${ }^{3}$ Family Health Unit Grão Vasco, Portugal
}

Received: 制 August 01, 2018; Published: 此 August 13, 2018

*Corresponding author: Nélio Veiga, Health Sciences Institute, Universidade Católica Portuguesa, Estrada da Circunvalação, 3504505, Viseu, Portugal

\begin{abstract}
The diabetic patient is subjected to a series of systemic complications and is more prone to develop other types of pathologies. This is equally applied to the oral cavity. This revision article aims to analyze some of the possible oral manifestations and complication derived from diabetes Concomitantly it is also pretended to know why these complications and more related to these patients, as well as to observe the differences between the pathogenesis and severity of oral pathologies among diabetics and non-diabetics.
\end{abstract}

Keywords: Diabetes; Oral Lesions; Periodontitis; Oral Candidiasis; Dental Caries; Oral Cancer

\section{Introduction}

Diabetes is a systemic disease belonging to the group of metabolic diseases in which there are high blood glucose levels. There are several types of diabetes: type 1, type 2, gestational and other types of diabetes [1]. The type 1 or insulin-dependent diabetic patients most often reaches children or young people and may also appear in adults and even among the elderly. This type is one of the rarest. In Type 1 Diabetes, pancreas $ß$ cells fail to produce insulin due to the massive destruction of insulin-producing cells. However, the causes of type 1 diabetes are not yet fully understood. These patients require lifelong insulin therapy because their body (pancreas) no longer has the power to make it. The type 2 diabetic patient has an insulin deficit and resistance to insulin, which implies a greater amount of insulin for the same amount of glucose in the blood. For this reason, patients with higher insulin resistance may, at an early stage, present higher insulin values and normal glucose values. Unlike type 1 diabetes, type 2 diabetes is related to poor eating habits as well as genetic factors [2].

Gestational diabetes appears during pregnancy in a previously non-diabetic patient and disappears after the end of gestation. However, this type of diabetes should not be underestimated since almost half of pregnant women with diabetes will later become people with type 2 diabetes if preventive measures are not considered. This type of diabetes can lead to several complications in childbirth such as the need for a cesarean section, an overweight baby and even spontaneous abortions [1,2]. Patients with Diabetes
Mellitus are more predisposed to oral candidiasis and xerostomia. However, the systemic pathology of which they are carriers can lead to the worsening of other oral conditions [2]. Throughout this revision article will address several oral manifestations erived or aggravated by diabetes. A more concrete analysis of the relationship between diabetes and some oral pathologies, namely oral candidiasis [3], dental caries [4], periodontitis [5], and premalignant lesions [6] will also be done.

\section{Materials and Methods}

To carry out this review article the search strategies included electronic databases, such as PubMed, Cochrane Library and Science Direct, reference lists of articles, and selected textbooks. Articles and textbooks used in this study were mainly reached by using the following keywords:" diabetes", "oral lesions", "periodontitis", "oral candidiasis", "dental caries", "oral cancer", "xerostomia”. All the articles and other literature analyzed had as exclusion criteria dating to 2000 and addressing prevalent oral pathologies in diabetic patients. Using these criteria, a total of 122 articles were obtained. After analyzing the abstracts and abstracts of each one, 22 articles were selected for analysis.

\section{Results/Discussion}

\section{Diabetes Oral Manifestations}

From the outset, it can be inferred that people with diabetes (either type 1 or type 2 ) have a significantly higher propensity to 
develop oral diseases compared to healthy people. This difference in prevalence comes from, among other factors, the vascular and neuropathic alterations that come with diabetes [8]. It is important to highlight the higher prevalence of oral lesions in type 2 diabetics than in type 1 diabetics [7]. In diabetic patients, the most common oral lesions are of the ulcerative type [7], and in type 2 diabetics the frequency of them is even greater. These lesions may lead to symptomatology such as glossodynia, discomfort and burning. In severe cases, it may affect everyday tasks, such as dysgeusia, oral paraesthesia [9], increased susceptibility to dental abscesses and reduced tissue regenerative capacity [10]. Other conditions found in diabetics such as angular cheilitis and hairy tongue may increase the propensity to manifest infections such as oral candidiasis [7]. Similarly, lower salivary flow (also termed xerostomia) observed in diabetics may lead to an increased risk of infections, since buffering ability and oral $\mathrm{pH}$ control is significantly affected $[4,8]$. Consequently, it is possible to feel dryness, diffuse erythema and stomatitis. In patients using removable prostheses, these problems are of even greater importance, considering that the lower salivary flow implies a much lower retention of the prosthesis [8].

Because of this, a higher prevalence of cases of prosthetic stomatitis in diabetics using removable prostheses against those who do not use is expected [11]. The presence of actinic cheilitis is also common [7], being more prevalent in the elderly population. The influence of diabetes on neoplastic development is also demonstrated due to hyperinsulinemia, hyperglycemia or chronic inflammation arising from it. This is explained by the high levels of glucose insulin common in diabetics and which give greater propensity to cell proliferation and creation of metastases [12]. Adding weight to this consideration is the fact that pre-malignant lesions such as leukoplakias and erythroplasias have a considerably higher prevalence in people who do not suffer from diabetes [13]. In view of the fact that some of these lesions are at high risk of malignancy, the proper control and inspection of diabetic patients is imperative $[13,14]$.

\section{Oral Candidiasis}

Candidiasis is the most common mycosis in the oral cavity, both in healthy patients and immunocompromised patients [15]. It is a superficial opportunistic infection caused by the fungus Candida spp. and is therefore more prone to arise due to several local and systemic factors [16]. And, in fact, the isolation of Candida species in most of the sample of diabetic patients has been studied. In diabetics, the type of diabetes and duration of the disease, as well as the degree of glycemic control are all associated with the presence of Candida [16]. In addition, in uncontrolled diabetics the rate of growth of Candida is higher than in healthy and diabetic patients [16-18]. The mechanism of higher susceptibility to Candida infection when a patient is diabetic is still not well established, however it is recognized that high levels of glucose in saliva (normal in diabetics), favors fungal proliferation [16]. The adhesion of fermentation products is determined with an essential first step in the colonization process and consequent infection by Candida.

High glucose levels in saliva can cause glycosylation products with proteins during peaks of hyperglycemia. These products may eventually lead to a larger number of receptors available for Candida. Thus, the initial mechanism of colonization is more likely to occur in diabetics and to occur with greater intensity [16]. The high prevalence of oral candidiasis is also associated in diabetics who use continuous acrylic removable prostheses [16], Candida albicans [17]. The acrylic used in prostheses can act as an additional field for adhesion of bacterial and fungal biofilm, which can be considerably thicker and lead to proliferation in other regions of the oral cavity. This is even more significant in diabetic patients who use prostheses and are older than 60 years [17]. In addition, diabetic patients with traumatic injuries due to the prosthesis have a lower resistance to infections [16]. Consequently, the epithelial permeability to bacteria and fungi is highly enhanced. It has also been proven that diabetic patients with smoking habits have higher blood glucose levels as a result of the effects of tobacco on adrenaline levels. Consequently, a higher Candida size among smokers compared to non-smokers would be expected [16]. Candida albicans is the most prevalent species of Candida species, both in diabetics and healthy people, although the percentage of cases is significantly higher [17]. Therefore, the epidemiology of Candida in the oral cavity in diabetic patients is quite complex, resulting from the combination of several different predisposers. Considering the presence of xerostomia in patients who use prostheses continuously and in diabetics, it is possible to establish a relationship between the presence of diabetes and prosthetic stomatitis associated with Candida [17]. However, it may not only come from the condition of hyperglycemia, so more research to clarify is necessary [9]. In conclusion, diabetic patients should maintain oral hygiene conditions to the maximum and remove the prosthesis overnight, to overcome the higher propensity for oral candidiasis infections [17].

\section{Dental Caries}

Taking the above information, glucose levels in the saliva of diabetics are higher than normal. As such, salivary $\mathrm{pH}$ changes are expected in these patients [4]. This reduction of $\mathrm{pH}$ will lead to a highly cariogenic environment, and consequently a higher activity of cariogenic bacteria will appear, namely Treponema denticola, Prevotella nigrescens, several species of Streptococci, among others [19]. The proliferation of these species will lead to an even lower $\mathrm{pH}$, and this vicious cycle continues, as well as affect the growth of protective microflora of the oral cavity [4]. When analyzing some particular types of diabetes, which is observed in type 1 diabetes, there is no significant difference in the number of dental caries between type 1 diabetics and healthy patients [20], when they are treated and medicated properly. Despite the higher frequency of meals by this group, the lower consumption of carbohydrates and a greater concern with health status may be behind this lack of correlation [20]. However, in type 2 diabetes there is a correlation with a significantly higher presence of dental caries [19] and worse dental health assessment scores, even worse when it is not controlled. This relationship may arise from two conditions: first, the greater propensity for periodontal disease to which diabetics are subjected, further exposing dental pieces and, on the other hand, the oral environment considerably more cariogenic in diabetics [19]. 


\section{Periodontitis}

The prevalence and severity of periodontal disease, as well as a greater propensity for its onset, are also evaluated by assessing type 2 diabetes. The strength of association between periodontitis and type 2 diabetes appears, however, to differ geographically, associated with factors such as genetics, cultural and ethnic differences [21]. The existence of a causal bilateral relationship is peculiar, since the increased severity of one pathology (diabetes or periodontitis) will negatively influence the severity of the other On the other hand, the improvement of glycemic control in type 2 diabetics is evaluated when their periodontal disease is treated [21]. Two theories are proposed regarding the mechanisms behind the bilateral association between type 2 diabetes and periodontal disease. The first hypothesis lies in altered immune changes in periodontal tissue. Diabetic patients tend to have vascular damage in the periodontium, leading to poor transport of nutrients and oxygen and less rapid elimination of metabolites. In addition, collagen metabolism is also affected, reducing the regenerative capacity of the periodontium. Finally, hyperglycemia leads to the formation of proteins called advanced glycolization end products, molecules associated with increased oxidative stress and an amplified inflammatory response [21].

The second hypothesis of association between type 2 diabetes and periodontal disease addresses periodontitis as an initial and exacerbating factor of type 2 diabetes. Substances from the common exaggerated inflammation response in periodontal disease may have a weight in controlling blood glucose levels, even having a directly proportional relationship [21]. This association can take on very serious levels in the diabetic patient and with periodontal disease, with authors going as far as to point out the association of chronic inflammation of periodontal disease with the onset of atherosclerosis, an equally possible condition in diabetics [22]. Therefore, it is essential for the diabetic patient to be aware of their increased propensity for the onset and rapid progression of periodontal disease, and to treat it as soon as signs and/or symptomatology appears. In fact, treatment of periodontal disease in uncontrolled diabetics can be relatively successful, with marked improvements after three months of treatment including mechanical periodontal therapy and adjuvant antimicrobial treatment [23]. Adequate periodontal treatment in the diabetic patient will also lead to higher quality and less morbidity in systemic terms [24].

\section{Oral Cancer}

There are currently some authors who affirm the relationship between diabetes (especially when poorly controlled) and a higher presence of pre-malignant lesions and tumors in the oral cavity [6]. However, the lack of comprehensive studies with good inclusion criteria, as well as a study that concludes a non-association between variables leaves the issue still controversial [6,11]. However, in diabetic patients with smoking habits the greater predisposition to the appearance of pre-malignant lesions, such as leukoplakia, is accepted [6]. In any case, the explanation given for a possible association between diabetes and the appearance of pre-malignant lesions (and an increased risk of cancer) comes from the higher production of reactive oxygen species and peroxides in diabetics, especially those with decompensated pathology and with excessive levels of triglycerides [6]. In turn, this will lead to a higher free radical presence, which may alter the DNA structure of the cells of the oral cavity, ultimately resulting in an increased risk of developing malignant lesions [6].

\section{Conclusion}

Diabetes is a disease with implications throughout the body, and the oral cavity is no exception. Several oral pathologies have a significantly increased risk of developing in diabetics, and even oral pathology may contribute to an increase in the severity of diabetes. It is also found that diabetics with poor glycemic control incur an even greater risk, both in terms of frequency and in terms of severity of oral pathologies. Although some oral pathologies do not yet have a proven association with diabetes, the diabetic patient should be aware of the increased risk of developing oral diseases. The diabetic patient should take extra care with oral hygiene and should have a dental appointment more frequently and be advised to solve or at least prevent a worse severity of oral pathology, thus contributing to a greater well-being and quality of life.

\section{References}

1. (2017) Portuguese Diabetic Association.

2. Mauri Obradors E, Estrugos Devesa A, Jané Salas E, Viñas M, López López J (2017) Oral manifestations of Diabetes Mellitus: a systematic review. Med Oral Patol Oral Cir Bucal 22(5): e586-e594.

3. Guggenheimer J, Moore PA, Rossie K, Myers D, Mongelluzzo MB (2000) Insulin-dependent diabetes mellitus and oral soft tissue pathologies. II. Prevalence and characteristics of Candida and candidal lesions. Oral Surgery, Oral Medicine, Oral Pathology, Oral Radiology, and Endodontology 89(5): 570-576.

4. Seethalakshmi C, Reddy R, Asifa N, Prabhu S (2016) Correlation of Salivary $\mathrm{pH}$, Incidence of Dental Caries and Periodontal Status in Diabetes Mellitus Patients: A Cross-sectional Study. J Clin Diagn Res 10(3): 4-12.

5. Faria AR, Morado PM, Cristina L, Inês F, Patrícia S, et al. (2006) Associação entre doença periodontal e patologias sistémicas. Rev Port Med Geral e Familiar 22(3): 379-382.

6. Auluck A (2007) Diabetes Mellitus: An Emerging Risk Factor for Oral Cancer? J Can Dent Assoc 73(6): 501-503.

7. Silva M, Barbosa K, Pereira J, Bento P, Godoy G, et al. (2015) Prevalence of oral mucosal lesions among patients with diabetes mellitus types 1 and 2. an Bras Dermato 90(1): 49-53.

8. Lima D, Nakata G, Balducci I, Almeida J (2008) Oral manifestations of diabetes mellitus on complete denture wearers. The Journal of Prosthetic Dentistry 99(1): 60-65.

9. Martinez R, Jaimes Aveldañez A, Hernández Pérez F, Arenas R, Miguel G (2013) Oral Candida spp carriers: its prevalence in patients with type 2 diabetes mellitus. An Bras Dermatol 88(2): 222-225.

10. Kakoei S, Hosseini B, Haghdoost A, Sanjari M, Gholamhosseinian A, et al. (2015) Evaluation of Salivary Secretory Immunoglobulin A Levels in Diabetic Patients and Association with Oral and Dental Manifestations. Sultan Qaboos Univ Med J 15(4): 507-511.

11. Al Maweri S, Ismail N, Ismail A, Al Ghashm A (2013) Prevalence of oral mucosal lesions in patients with type 2 diabetes attending hospital universiti sains malaysia. Malays J Med Sci 20(4): 39-46. 
12. Li S, Lee YC, Li Q Chen CJ, Hsu WL, et al. (2015) Oral lesions, chronic diseases and the risk of head and neck cancer. Oral Oncol 51(12): 10821087.

13. Dikshit R, Ramadas K, Hashibe M, Thomas G, Somanathan T, et al. (2006) Association between diabetes mellitus and pre-malignant oral diseases: a cross sectional study in Kerala, India. Int J Cancer 118(2): 453-457.

14. Malvania E, Sheth S, Sharma A, Mansuri S, Shaikh F, et al. (2016) Dental caries prevalence among type II diabetic and nondiabetic adults attending a hospital. J Int Soc Prev Community Dent 6(3): S232-S236.

15. Manfredi M, McCullough M, Al Karaawi Z, Vescovi P, Porter S (2006) Analysis of the strain relatedness of oral Candida albicans in patients with diabetes mellitus using polymerase chain reaction-fingerprinting. Oral Microbiol Immunol 21(6): 353-359.

16. Soysa N, Samaranayake L, Ellepola A (2006) Diabetes mellitus as a contributory factor in oral candidosis. Diabet Med 23(5): 455-459.

17. Belazi M, Velegraki A, Fleva A, Gidarakou I, Papanaum L, et al. (2005) Candidal overgrowth in diabetic patients: potential predisposing factors. Mycoses 48(3): 192-196.

18. Huang J, Liu Y, Liu H (2012) Comparative study on oral candidal infection in individuals with diabetes mellitus and impaired glucose regulation. Zhonghua Kou Qiang Yi Xue Za Zhi 47(6): 335-339.

ISSN: 2574-1241

DOI: 10.26717/BJSTR.2018.07.001574

Nélio Veiga. Biomed J Sci \& Tech Res

(C) (i) This work is licensed under Creative

Submission Link: https://biomedres.us/submit-manuscript.php
19. Satoru Y, Masato U, Shunichi M (2017) Association between numbers of decayed teeth and HbA1c in Japanese patients with type 2 diabetes mellitus. Ups J Med Sci 122(2): 108-113.

20. Garcia R, Coelho A, Paula A, Marques M, Caramelo F, et al. (2016) Prevalence of Dental Caries in Type 1 Diabetic Patients Treated with Insulin Pump. Acta Med Port 29(7-8): 461-467.

21. Susanto H, Nesse W, Dijkstra P, Agustina D, Vissink A, et al. (2011) Periodontitis prevalence and severity in Indonesians with type 2 diabetes. J Periodontol 82(4): 550-557.

22. Napora M, Grabowska E, Górska R (2016) Prospective Analysis of the Relationship Between the State of Periodontal Tissues and Changes in Selected Cardiovascular Parameters in Patients with Type 2 Diabetes. Adv Clin Exp Med 25(5): 879-886.

23. Promsudthi A, Pimapansri S, Deerochanawong C, Kanchanavasita W (2005) The effect of periodontal therapy on uncontrolled type 2. Oral Dis 11(5): 293-298.

24. Shangase S, Mohangi G, Hassam Essa S, Wood N (2013) The association between periodontitis and systemic health: an overview. SADJ 68(1): 1012.

BIOMEDICAL
RESEARCHES $\quad \begin{aligned} & \text { Assets of Publishing with us } \\ & \text { - Global archiving of articles }\end{aligned}$

